\title{
Supporting Research with Weblogs: A Study on Web-based Research Support Systems
}

\author{
JingTao Yao \\ Department of Computer Science \\ University of Regina, Regina, Canada \\ jtyao@cs.uregina.ca
}

\begin{abstract}
Research environments have been changing with the availability of new technologies for decades. Researchers are benefited from digital libraries, online databases, and Web search engines recently. It is expected that the Webbased research systems play an important role in supporting research activities. Blogs, a new form of mainstream personal communication and a new voice for traditional media, recently gained much attention from the public, industrial and academic. This article studies issues on Web-based research support systems with blogs.
\end{abstract}

\section{Introduction}

As new technologies evolve and existing technologies expand, scientists need to adjust accordingly to make full use of technologies when carrying out research. Scientists and researchers face challenges in using online information resources, such as information overload, misinformation, fees, as well as poorly designed navigation, retrieval, and browsing tools. Supporting scientists and researchers to meet such challenges remains an important issue. The study of Web-based research support systems (WRSS) aims to provide a common framework in supporting some research activities with the Web technology [9, 18, 19]. WRSS is a particular type of Web-based support systems [13, 15, 17]. Web-based Support Systems (WSS) is viewed as a multidisciplinary research that focuses on supporting human activities in various domains and fields based on computer science, information technology, and Web technology. The study of WSS aims to provide support to all kinds of human activities in a standard Web platform. WSS provide distributed support with no restrictions on geography location or time. Examples of WSS are available from proceedings of previous WSS workshops [13, 15].

Weblogs (or blogs, we will use Weblog and blog inter- changeable in this article), as a new form of mainstream personal communication and a new voice for traditional media, have been recently gained much attention from the public, industrial and academic. It is feasible to adopt Blog mechanism in a Web-based research support systems. Researchers within a research group or scientists in same research domain may share their views via blog publications and comments. Blogs now acts as a new communication channel. They can also record history and evolution of research ideas to enhance research quality and productivity. This position paper tries to brief issues on Web-based research support systems with blogs. It is not our intention to present a completed system and to cover all aspects of Weblogs in support of Web-based research support systems in this article. The aim of this paper is to bring people's attention on study related issues.

\section{Weblogs}

Blogs offer a new way for general public to express their opinion in contract traditional one-way street media [7]. They are a special type of website that publish information in the format of journal or diary. The contents are often displayed in reverse chronological order. As of September 2006, Technorati (www.technorati.com), a Weblog-tracking company, tracks 55.4 million blogs where the figure indicated in Rosenbloom's article was only 4.2 millions in October 2004 [7]. According to Technorati, there are approximately 75,000 new blogs a day and around 50,000 blog updates an hour. A blog normally contains text, images, hyperlinks and some times audio or video clips.

Additionally, a similar technique, the Wikipedia (www.wikipedia.com), also know as the online encyclopedia, has also gained popular due to it's openness to public. Originated in 2001, there were over 1.41 million English entries and over 5 million entries with various languages according reports on the Wikipedia's Web pages in September 2006. 
We may conclude from the above figures that Weblogs and the Wikipedia are well accepted by general public. The rapid development blog has also attracted researchers in different domains. Oravec suggested that educators could use weblogs to encourage students to access the Internet for useful information and students could use weblogs to help them in organizing the resources they locate [6]. Divitini et al. reported a study on using a blog to support teacher education. The system had been used to support communication and reflection while students were spending a period of teaching practice [1].

Du et al. claimed that blog success was associated with the type of blogging tool used since technology characteristics affect the presentation and organization of blog content as well as social interaction between bloggers. Indeed, the Wiki technology has been used as a tool to discuss issue on organizing academic conferences [3]. Wagner considered the Wiki as a technology for conversational knowledge management and group collaboration [10].

\section{Research Activities and Web-based Research Support Systems}

Similar to other Web-based support systems, WRSS is also interdisciplinary. It involves at least three domain of studies: research methodologies (purpose of research, research methods, research activities), computer science (computer systems that support various research activities), and the Web (as an infrastructure and a medium of support delivery, as a common user interface). Many computerized systems, although not designed specifically for research support, have been used by scientists in different stages of research. Web-based research support systems aim at pooling together all these isolated efforts and unintegrated systems with a shared goal of research support.

One of the key issues on Web-based research support systems is to study research activities. Research activities can be broadly classified into two levels, the institutional level and the individual level [19]. The institutional level deals with the management of research and research projects in an institution [9]. The individual level is the actual research process of a scientist. A research process model at individual level may include the following phases: idea generating, problem definition, procedure design and planning, observation and experimentation, data analysis, results interpretation and explanation, communication and dissemination [18]. It is possible to combine several phases into one, or to divide one phase into more detailed steps. Moreover, the research process does not follow a rigid sequencing of the phases. Iteration of different phrases may be necessary.

A WRSS should consist of many sub-systems to support various research activities. A common database and knowl- edge bases may be shared amongst sub-systems. As one cannot have a clear classification of research activities, it is difficult to have a clear classification of different types of support sub-systems. Yao has summarized some supporting functionalities as exploring support, retrieval support, reading support, analyzing support, and writing support [18]. We will represent the main results in this section.

Exploring support: Exploration is probably the very first stage of research activity and it plays an important role. The main activities of exploration include browsing databases, libraries, and the Web. When a scientist have a vague idea and would like to confirm or clarify it, he or she may browse the literature in order to find what other fellow researchers are doing. If the Web is used for browsing, historical data can be tracked and analyzed by using machine learning and data mining tools to provide a scientist useful information and hints. Web browsers are one of useful exploration tools. Their functions need to be expanded for providing research support. For instance, agent technology is included in WRSS in order to support researchers to find related information.

Retrieval support: Once a relatively solid idea is formed, it is necessary to conduct literature review to find relevant information. Retrieval support assists retrieval related activities, such as browsing, searching, organization, and utilization of information. ISI's Web of Science (http://portal.isiknowledge.com/portal.cgi) includes a find related record feature. The level of "relationship" is measured by the number of co-cited papers. Similar research papers could be retrieved if they share some common references. In fact, references may be used as one of criteria to cluster or classify research papers.

Reading support: Every researcher expects other researchers to read his/her research publications. Reading critically and extensively is crucial, especially in the preparation stage. The advances in digital libraries and electronic publications make reading and its support possible. Readers can add bookmarks, make notes, link different parts of an article, and make logical connections of different articles. One of the key tasks for reading support sub-system is to assist a reader in actively finding relevant materials and constructing cognitive maps based on the materials read. Online dictionaries may also be useful in reading support especially in reading documents written in foreign languages [5].

Machine learning, text mining methods and agent technologies can be used to assist a reader by learning from the reading history. and to actively look for useful information and periodically inform scientists with new information.

Analyzing support: Data analyzing is an important step for many research projects. Graphics and visualization are certainly useful tools in supporting data analysis. Although there are many tools available for choosing, one of a key 
roles for analyzing support is to assist scientists to find and use the right tool for a particular problem in analyzing data. Tool management is also an essential component. If the functions of tools are described as plain text, information retrieval systems can be used to find the right tool.

Writing support: Writing support sub-systems may be considered as first generation of support systems. Researchers have been using various word-processors and typesetting software for decades. For example, Microsoft Word and Latex are two of the most popular used tools in supporting scientific writing. It is important to assist a scientist on typesetting since $50 \%$ of time mey be spent to typeset a paper as stated in Schulman's satire [8]. Many tools come with additional functions, such as spellingchecking, grammar-checking, and a range of other agents. One of the teaching component in graduate school is on academic writing. A writing support subsystem may guide a writer in writing by providing various article format. It can also assist a writer to find some redundant phrases that appear in carelessly written papers. Furthermore, a writing support system may find relevant articles based on the text written by a scientist and suggests possible references.

Dissemination support: Funding agencies may not consider a research process completed until the results are validated and transmitted to an appropriate target audience. One of the most common and effective means of disseminating results is through refereed publications. Paper submission, reviewing, and publication processes are incredibly time consuming and difficult to manage without the help of computer systems along with the Internet. Many dissemination support products are developed and being used recently. For instance, Cyberchair (www.cyberchair.org), established in 1996, claims it as "a free Web-based paper submission and reviewing system with PC [(Program Committee)] meeting and proceedings preparation support". It has been adopted by many conference organizers.

Web-based research support systems aims to assist scientists in improving their research quality and productivity. The study of Web-based support systems and research methodology makes the study, development, usage and evaluation of such systems feasible.

\section{The Role of Weblogs}

Weblogs provide an excellent new channel for research discussion, communication, and collaboration. Some people may confuse Weblogs with Web pages. As we discussed above, blogs are essentially a special type of Web pages. In addition to the diary type format, one of the key differences is that readers may add comments on a particular blog entry. This highlights an important feature for research discussion and communication and could be beneficial to research support systems. We would like to study the role of blogs on research support in this section.

A Web-based research support system may gain from the diary feature in blogs to support researchers' daily activities. A research diary is normally a record of the researcher's involvement in a project [4]. In the digital age, researchers write their diary or journal with all kinds of new electronic devices, such as PDA, mobile phone, digital recorder, to just name a few. One of the purposes of research diary is to generate a history of the project, the researcher's thinking and the research process. It is expected that a WRSS will provide a summary mechanism for recorded diary of a project. A researcher will be able to trace the idea evolution and history, which in turn benefits the future research. Comments by team members or peers in same research domain will assist a researcher to clarify the ideas and brainstorm.

Blogs may also provide a research forum for a team project. Team members post their diaries to blog though WRSS. Security measures may be applied to grant team members, peers and general public different levels of access to the blogs. In certain stages, or for certain contents, only limited people have blog information accessing rights. Not every reader is granted viewing, submitting or altering rights. This will guarantee information sharing with a research team and prevention of information leaking. Most blog software provide trackback and pingback features in order to trace a Weblog or a site when a new post is added. This will ensure that new comments are dealt accordingly. In order to avoid flood of hatred or spam comments, spam filters are often included. For instance, only logged in users are allowed to post or comment. Moderation and delay are also useful in preventing comment spam.

Blogs provide a new dissemination channel for research results. With blogs, not only completed research results, but also half baked ideas will be delivered. There is no restriction on the time, the devices used, and the geographic location for other researchers to gain such information. In addition, people make comment on ideas, thoughts, research methods, and research results. If traditional reviewing processes is considered, a project could be reviewed and received feedback from peers at very beginning.

Cite or reference other researchers' work is a norm in scientific publications. It is essential to provide complete citation information in benefit readers to retrieve cited articles. Hyperlinks on blogs serve the exact same function. The reference papers are just a click away on blogs. Furthermore, the hyperlinks on blogs also lead to ideas, comments, historical records, discussions, as well other blogs. Hyperlink management and visualizing link network will help researchers to gain more understanding on idea generation and evolution. Drill down, thumbnail, and expansible tree allow users to easily browse blog contents with their own interests and paces. 
Blog data management include organizing, classifying, backup, and retrieving blog contents. Blogs may be arranged either in chronological order or reverse chronological order. It is useful to organize blogs by owners, topics, or their popularity. Analyzing and visualizing of blog classifications provide hints and clues for research development. Blog contents search and query are useful especially for larger blogs. Commercial or in house search engines play different roles. Popular search engines such as Blogdigger (www.blogdigger.com), Feedster (www.feedster.com), and Technorati search all blog contents on the Internet. Search engines as a sub-system for WRSS provide content search within a personal or group blog space.

\section{Conclusion}

We introduce Web-based research support systems in the aim of assisting quality research in the Web age. After briefing research related activities, some key functionalities of WRSS are summarized. Weblogs as a special type of Web pages and a new publication media have shown a rapid development in just a few years. We present some blog features that support research activities.

\section{References}

[1] M. Divitini, O. Haugalokken, and E. M. Morken, Blog to Support Learning in the Field: Lessons Learned from a Fiasco, Proceedings of the 5th IEEE International Conference on Advanced Learning Technologies (ICALT'05), 2005, pp219-221.

[2] H.S. Du and C. Wagnera, Weblog success: Exploring the role of technology, International Journal of HumanComputer Studies 64(9), 789-798, 2006.

[3] M.D. Hill, J.-L. Gaudiot, M. Hall, J. Marks, P. Prinetto, and D. Baglio, A Wiki for Discussing and Promoting Best Practices in Research, Communications of the ACM, 49(9), 6364, 2006.

[4] I. Hughes, How to Keep a Research Diary, http://www.scu.edu.au/schools/gcm/ar/arr/arow/rdiary.html, 1996.

[5] H. Mochizuki and A. Tera, Constructing Web-Based Japanese Text Reading Support System and its Evaluation, Proceedings of the International Conference on Computers in Education, 2002, pp1478-1479.

[6] J.A. Oravec, Bookmarking the World: Weblog Applications in Education, Journal of Adolescent \& Adult Literacy, 45(7), 616-621, 2002.

[7] A. Rosenbllom, The Blogosphere, Communications of ACM, 47(12), 31-33, 2004.
[8] E.R. Schulman, How to Write a Scientific Paper, Annals of Improbable Research, 2(5), 8, 1996.

[9] H. Tang, Y. Wu, J.T. Yao, G.Y. Wang, and Y.Y. Yao, CUPTRSS: A Web-based Research Support System, Proceedings of the Workshop on Applications, Products and Services of Web-based Support Systems (WSS'03), Halifax, Canada, Oct 13, 2003, pp21-28.

[10] C. Wagner, Wiki: A Technology For Conversational Knowledge Management And Group collaboration, Communications of the Association for Information Systems, 13, 265289, 2004.

[11] J.T. Yao, Design of Web-based Support Systems, 8th International Conference on Computer Science and Informatics (CSI'05), Salt Lake City, USA July 21-26, 2005, pp349-352.

[12] J.T. Yao, On Web-based Support Systems, Proceedings of the 2nd Indian International Conference on Artificial Intelligence, Pune, India, December 20-22, 2005, pp2589-2600.

[13] J.T. Yao and P. Lingras, Proceedings of 2003 WI/IAT Workshop on Applications, Products and Services of Web-based Support System (WSS'03), Halifax, Canada, Oct 13, 2003.

[14] J.T. Yao, W.-N. Liu, L. Fan, Y.Y. Yao and X.D. Yang, Supporting Sustainable Communities with Web-based Information Systems, Journal of Environmental Informatics, 7(2), 84-94, 2006.

[15] J.T. Yao, V.V. Raghvan and G.Y. Wang, Proceedings of the Second International Workshop on Web-based Support System (WSS'04), Beijing, China, September 20, 2004.

[16] J. T. Yao and Y.Y. Yao, Web-based Information Retrieval Support System s: building research tools for scientists in the new information age, Proceedings of the IEEE/WIC International Conference on Web Intelligence (WI'03), Halifax, Canada, Oct 13-17, 2003, pp570-573.

[17] J.T. Yao and Y.Y. Yao, Web-based Support Systems, Proceedings of the Workshop on Applications, Products and Services of Web-based Support Systems (WSS'03), Halifax, Canada, Oct 13, 2003, pp1-5.

[18] Y.Y. Yao, A Framework for Web-based Research Support Systems, Proceedings of the 27th Annual International Computer Software and Applications Conference (COMPSAC'03), Dallas, USA, November 3-6, 2003, IEEE Computer Society Press, pp601-606.

[19] Y.Y. Yao, Web-based research support systems, Proceedings of the Second International Workshop on Web-based Support Systems (WSS'04), September 20, 2004, Beijing, China, pp1-6. 